\title{
Síntesis de un nanomaterial magnético de plata para aplicaciones ambientales
}

\section{Synthesis of a silver magnetic nanomaterial for environmental applications}

\author{
Fabián Ricardo Soto González ${ }^{1}$, María Gisela Morales ${ }^{2}$, Adriana Mabel Pajares ${ }^{1,2}$, \\ Juan Pablo Escalada ${ }^{1}$ \\ fabi.soto11@hotmail.com,moralesmriagisela@yahoo.com.ar,apajares@live.com, \\ jescalada@uarg.unpa.edu.ar \\ ${ }^{1}$ Instituto de Tecnología Aplicada, UARG, Universidad Nacional de la Patagonia Austral- \\ UNPA, 9400 Río Gallegos, Argentina. \\ ${ }^{2}$ Departamento Ingeniería Química, FI, Universidad Nacional de la Patagonia San Juan \\ Bosco, 9000 Comodoro Rivadavia, Argentina.
}

Recibido: 15/04/2021. Aceptado: 15/11/2021

\section{RESUMEN}

El desarrollo de nuevos sensibilizadores para la degradación fotoquímica de contaminantes ha sido un foco de atención en los últimos años, es por ello que los nanomateriales han tomado una gran relevancia en este campo. En este contexto, se trabajó en la síntesis de un nuevo nanomaterial de óxido de hierro $\left(\mathrm{Fe}_{3} \mathrm{O}_{4}\right)$ y plata $(\mathrm{Ag})$, el cual fue obtenido en dos etapas; en primer lugar se sintetizaron las nanopartículas de magnetita (NPs) para luego adsorber plata sobre éstas obteniendo el nanocompuesto (NC) de $\mathrm{Fe}_{3} \mathrm{O}_{4} / \mathrm{Ag}$. Se evaluó su capacidad como fotosensibilizador en la fotodegradación aireada del alcohol furfurílico, donde se observó la degradación del mismo aunque con una baja eficiencia.

Palabras clave: Nanopartículas; Fotodegradación.

\begin{abstract}
The development of new sensitizers for the photochemical degradation of pollutants has been a focus of attention in recent years, which is why nanomaterials have become very important in this field. In this context, we worked on the synthesis of a new nanomaterial of iron oxide $\left(\mathrm{Fe}_{3} \mathrm{O}_{4}\right)$ and silver $(\mathrm{Ag})$, which was obtained in two stages; In the first place, the magnetite nanoparticles (NPs) were synthesized and then silver adsorbed on them, obtaining the $\mathrm{Fe}_{3} \mathrm{O}_{4} / \mathrm{Ag}$ nanocomposite (NC). Its ability as a photosensitizer in the aerated photodegradation of furfuryl alcohol was evaluated, where its degradation was observed, although with low efficiency.
\end{abstract}

Keywords: Nanoparticles; Photodegradation. 


\section{INTRODUCCIÓN}

Las acciones antropogénicas generan diferentes tipos de contaminación de las aguas superficiales. Uno de los procesos de depuración de los contaminantes es la fotodegradación. Esta consiste en la rotura de moléculas de compuestos indeseables por efecto de la luz. Dado que un gran número de contaminantes son incoloros y por ello incapaces de absorber significativamente la radiación lumínica natural solar que llega a la superficie de la tierra, nos hemos interesado particularmente en procesos fotosensibilizados, en los que una sustancia presente en el medio (fotosensibilizador) es la responsable de la absorción primaria de la luz solar. A partir de ese hecho pueden desencadenarse una serie de procesos fotoquímicos y fotofísicos que eventualmente terminan con la degradación del contaminante. Este trabajo se basó en la síntesis de un nanomaterial de óxido de hierro $\left(\mathrm{Fe}_{3} \mathrm{O}_{4}\right)$ y plata $(\mathrm{Ag})$ y en el estudio de sus propiedades en cuanto a la absorción de luz UV-visible y generación de especies reactivas de oxígeno para tener un panorama sobre su posible utilización como fotosensibilizador en procesos de fotodegradación de contaminantes.

\section{MARCO DE REFERENCIA}

Si bien en el siglo XIX Michael Faraday (1875) había descrito las propiedades ópticas de metales en escala nanométrica, es en las últimas décadas cuando estos materiales han tomado mayor relevancia por las innumerables aplicaciones que día a día surgen, tanto industriales, ópticas, electrónicas, biomédicas e incluso ambientales. La nanotecnología manipula la materia en la escala de los nanómetros, es decir $10^{-9} \mathrm{~m}$. Así las nanopartículas comprenden a aquellas partículas de tamaños entre $10^{-7}$ y $10^{-9} \mathrm{~m}$. (Horikoshi y Serpone, 2013). Sus múltiples aplicaciones modernas, se deben a que las nanopartículas metálicas tiene propiedades físicas y químicas que las diferencian de los metales a granel (Laurent et al., 2008). Por ejemplo, disminuye su punto de fusión, esto permite emplear pasta de nanopartículas de metales para imprimir las placas de los circuitos electrónicos. Sus propiedades ópticas dependen fuertemente del tamaño y forma de las partículas, lo cual les confiere distintos colores y por lo cual han encontrado aplicaciones como componentes de pinturas para distintos usos. Los avances recientes sugieren que varios problemas relacionados con la calidad del agua podrían resolverse o mejorarse considerablemente mediante el empleo de productos que surjan del desarrollo de la nanotecnología (Schulte y Dutta, 2005) (Liu et al., 2020). En los últimos años ha recibido especial atención el empleo de nanomateriales de hierro como adsorbente de compuestos orgánicos o metales pesados en la remediación de ambientes contaminados (Auffan et al., 2009) (Liu et al., 2021).

Durante más de dos décadas, las nanoestructuras híbridas han atraído la atención de los científicos en nanotecnología debido a sus aplicaciones multifuncionales únicas. Las nanoestructuras de $\mathrm{Ag} / \mathrm{Fe}_{3} \mathrm{O}_{4}$ son uno de los sistemas binarios más importantes. En los últimos años se ha estudiado su actividad como antibacterial y antimicrobial (Wang et al., 2021) (Chen et al., 2019).

Estos compuestos, capaces de absorber la luz visible, podrían ser utilizados como fotocatalizadores para la degradación de diferentes contaminantes ambientales.

Las reacciones fotoquímicas juegan un papel muy importante en el medioambiente. La activación fotoquímica es la principal fuerza conductora de la transformación de sustancias en la atmósfera y determina la degradación de compuestos que no se biodegradan de un modo eficaz en el agua (Boule y Bahnemann, 1999). 
La fotodegradación es una reacción química que se produce bajo la influencia de la luz; puede tener lugar en la atmósfera, en la superficie del suelo y del agua. El proceso de fotodegradación se inicia cuando la molécula absorbe un cuanto de energía (fotón) y, como resultado, se produce la rotura de enlaces químicos en la molécula. Esto conlleva a la formación de radicales muy reactivos. Sin embargo, el espectro de absorción de muchos contaminantes ambientales se extiende escasamente hasta los $320 \mathrm{~nm}$, de manera que estos sustratos, son prácticamente transparentes a la luz solar que alcanza la superficie terrestre. En las aguas superficiales, el mecanismo de fotodegradación tiene lugar tras la absorción de luz visible por un sensibilizador capaz de interactuar con el oxígeno disuelto generando especies reactivas de oxígeno (ROS).

El oxígeno molecular en su forma más estable $\left(\mathrm{O}_{2}\right.$, en esta forma se encuentra en el aire que nos rodea) se puede considerar como un radical libre pues tiene dos electrones no apareados con spins paralelos y es un potente agente oxidante, sin embargo para oxidar una molécula no radical y aceptar un par de electrones, estos deben de tener el mismo spin (paralelo) para ocupar los orbitales vacantes en el $\mathrm{O}_{2}$. Los electrones de un átomo o de una molécula no radical no cumplen con este criterio, puesto que tienen spin antiparalelo. Esta restricción de spin hace que el $\mathrm{O}_{2}$ acepte solo un electrón a la vez y explica por qué reacciona lentamente con la mayoría de las moléculas no radicales. Por el contrario, reacciona notablemente rápido con radicales por transferencia de un solo electrón (Halliwell, 2006).

Así, aunque el oxígeno molecular en su estado basal es poco reactivo, su reducción parcial genera EROs, tales como oxígeno singlete molecular $\mathrm{O}_{2}\left({ }^{1} \Delta_{\mathrm{g}}\right)$, anión radical superóxido $\left(\mathrm{O}_{2}{ }^{*}\right)$, radical hidroxilo $\left(\mathrm{HO}^{*}\right)$ y peróxido de hidrógeno $\left(\mathrm{H}_{2} \mathrm{O}_{2}\right)$, con características de entre moderadas a relativamente fuertes oxidantes.

Es sabido que un evento de fotosensibilización oxidativa, de aparentemente simple descripción global, puede ser muy complejo y a la hora de explicar la serie de procesos fotoquímicos/fotofísicos involucrados puede que no sea sencillo hacerlo en términos mecanísticos detallados.

El Esquema 1 representa, de manera muy simplificada, un proceso de fotooxidación sensibilizada, en solución, del sustrato Q, transparente a la luz. El sensibilizador (S) absorbe luz y genera estados electrónicamente excitados singlete $\left({ }^{1} \mathrm{~S}^{*}\right)$ y triplete $\left({ }^{3} \mathrm{~S}^{*}\right)$. Estos estados pueden ser desactivados por interacción con el sustrato (Q). La especie ${ }^{3} \mathrm{~S}^{*}$, de relativamente larga vida en solución, puede ser interceptada por oxígeno molecular disuelto en el medio $\left(\mathrm{O}_{2}\left({ }^{3} \Delta_{\mathrm{g}}{ }^{-}\right)\right)$, generando ROS que pueden reaccionar con $\mathrm{Q}$ e incluso con el propio $\mathrm{S}$ produciendo oxidación.

$$
S+h v \rightarrow 1 S^{*} \rightarrow 3 S^{*} O_{2}\left(3 \Delta_{g}^{-}\right) \rightarrow \text { ROS } Q ; S \rightarrow \text { Productos }
$$

Esquema 1. Secuencia simplificada de hipotéticos pasos involucrados en un proceso de oxidación fotosensibilizada.

\section{RESULTADOS ANÁLISIS Y DISCUSIÓN}

\subsection{Materiales y métodos}

\subsubsection{Materiales}

- Almidón soluble

- Cloruro férrico hexahidratado $\left(\mathrm{FeCl}_{3} \cdot 6 \mathrm{H}_{2} \mathrm{O}\right)$ 
- Nitrato de plata $\left(\mathrm{AgNO}_{3}\right)$

- Sulfato ferroso heptahidratado $\left(\mathrm{FeSO}_{4} \cdot 7 \mathrm{H}_{2} \mathrm{O}\right)$

- Amoníaco hidratado $\left(\mathrm{NH}_{3} \cdot \mathrm{H}_{2} \mathrm{O}\right)$

- Alcohol furfurílico

Todas las soluciones fueron preparadas en agua ultrapurificada de conductividad menor a 0,1 $\mu \mathrm{S} / \mathrm{cm}$

\subsubsection{Equipos}

- Espectrofotómetro Hewlett Packard 8453 (arreglo de diodos), con intervalo de detección 190 - $1100 \mathrm{~nm}$ (para tomar los espectros de absorción).

- Electrodo Orion 081010, específico para oxígeno disuelto (para consumo de oxígeno).

- Espectrofotómetro de absorción atómica Perkin Elmer AAnalyst 200 (para cuantificación de Ag y Fe).

- Fotolizadores LEDs construídos por el equipo de trabajo.

\subsection{Resultados y discusión}

\subsubsection{Síntesis}

La metodología utilizada en la síntesis de las nanopartículas fue una modificación de la descrita por Peter R. Chang et al. (2011).

Se prepararon $200 \mathrm{ml}$ de una solución de almidón $0,6 \%$ p/p pesando $1,21 \mathrm{~g}$ de almidón y llevándolo al volumen total de la solución. La misma se agitó con una barra magnética a 1000 rpm y simultáneamente se la sometió a una atmósfera de nitrógeno. Luego de 30 minutos se introdujeron $0,76 \mathrm{~g}$ de Sulfato Férrico Heptahidratado $\left(\mathrm{FeSO}_{4} .7 \mathrm{H}_{2} \mathrm{O}\right)$ y $1,49 \mathrm{~g}$ de Cloruro Férrico $\left(\mathrm{FeCl}_{3} \cdot 6 \mathrm{H}_{2} \mathrm{O}\right)$, se continuó agitando y burbujeando nitrógeno por 20 minutos (Fig. 1).

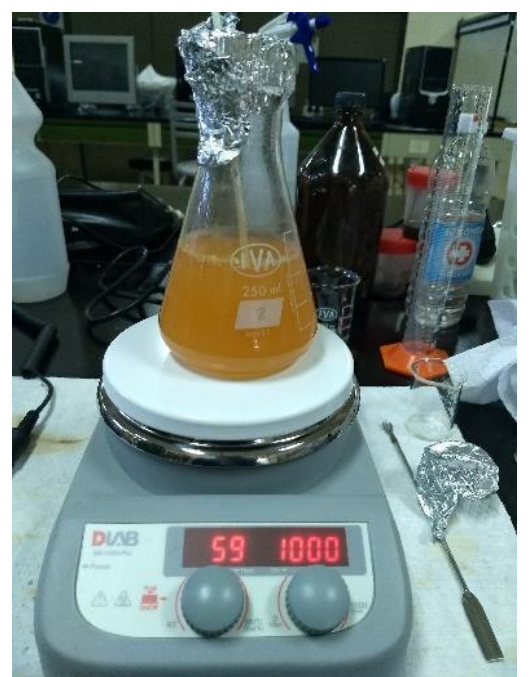

Fig. 1. Solución de almidón, cloruro férrico y sulfato férrico en atmósfera de nitrógeno

A fin de ajustar el pH de la solución a un valor de 10, se utilizaron aproximadamente $2 \mathrm{ml}$ de Hidróxido de Amonio $\left(\mathrm{NH}_{4} \mathrm{OH}\right)$ al $28 \%$ p/p. Se introdujo gota a gota el reactivo registrando el $\mathrm{pH}$ con cintas universales medidoras de $\mathrm{pH}$. Durante el proceso se observó un cambio de 
coloración al introducir los primeros ml de solución, virando de color naranja a marrón, a una coloración oscura al introducir los próximos ml, llegando finalmente a un pH de 10 (Fig. 2).
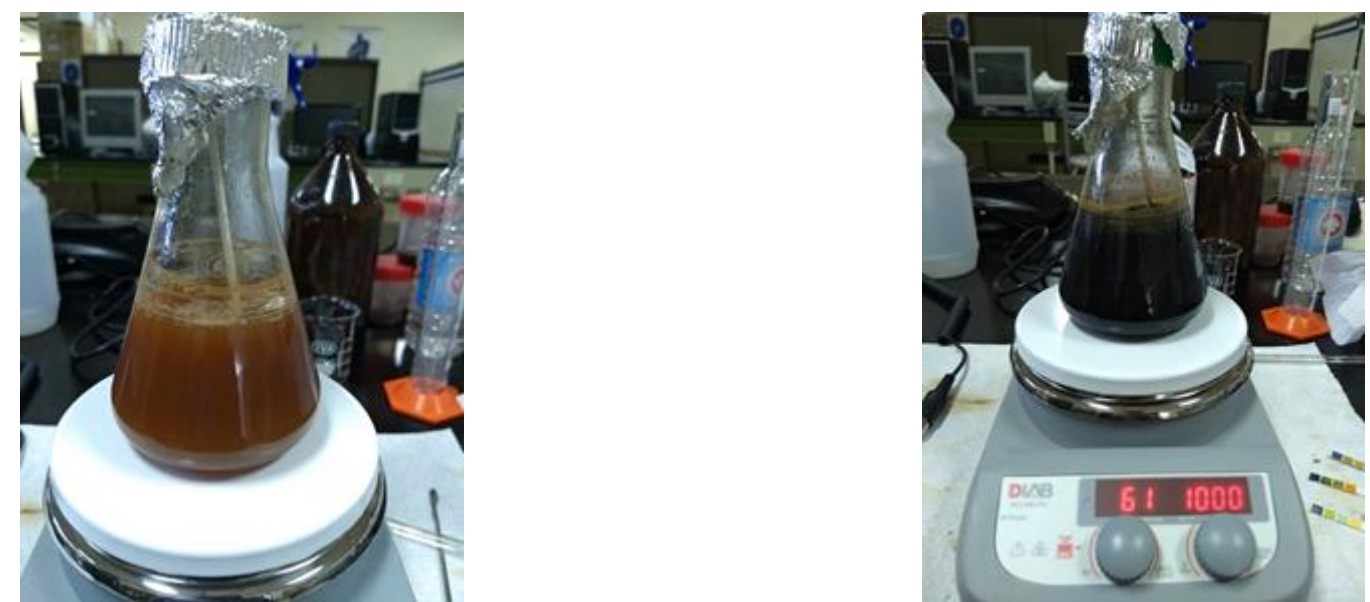

Fig. 2 Variación del color de la solución con el agregado de $\left(\mathrm{NH}_{4} \mathrm{OH}\right)$

Ajustado el pH se continuó con el proceso de calentamiento, agitación y burbujeo por 3 horas. Transcurrido este tiempo, se detuvo el sistema y se dejó enfriar a temperatura ambiente.
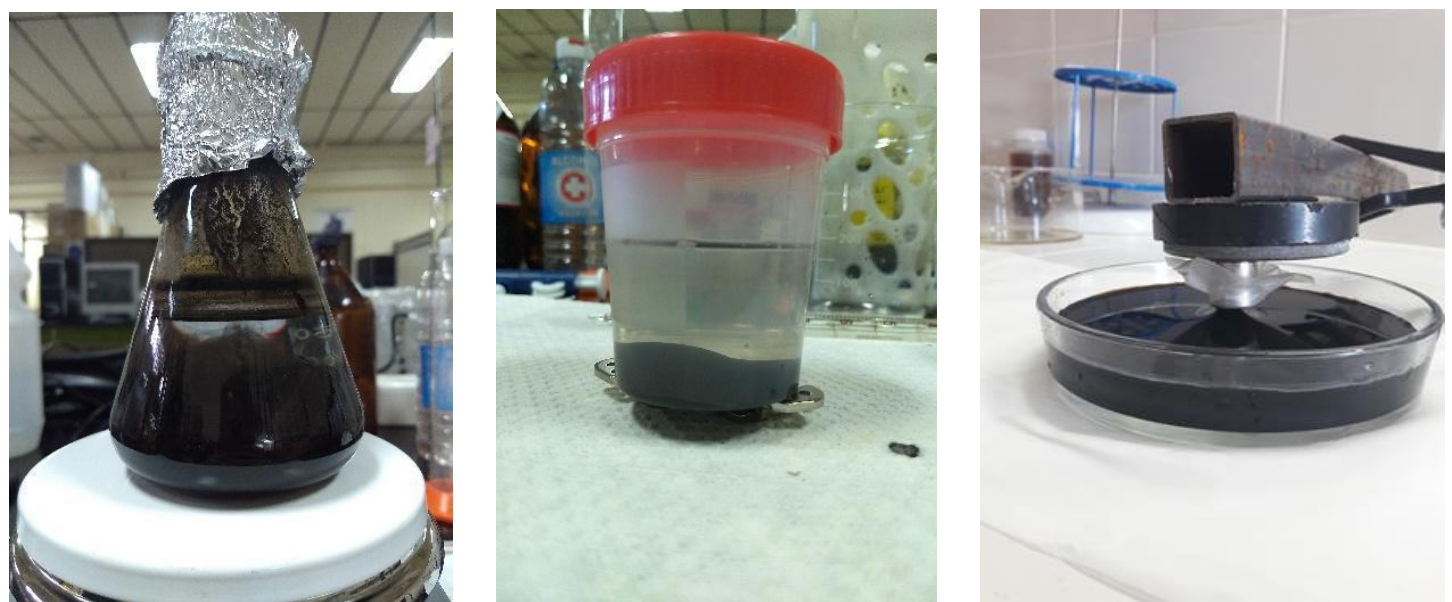

Fig. 3. Separación magnética de las NPs de $\mathrm{Fe}_{3} \mathrm{O}_{4}$

Una vez alcanzada la temperatura ambiente, se procedió a la separación de las NPs de $\mathrm{Fe}_{3} \mathrm{O}_{4}$ de la solución realizando sucesivos lavados con agua destilada y con la ayuda de un imán de neodimio. Tal como se observa en la Fig. 3, luego de este procedimiento se obtuvieron grandes cantidades de las NPs con importantes propiedades magnéticas.

La síntesis de nanopartículas de hierro se pudo realizar con éxito, obteniendo al final del proceso $1,5690 \mathrm{~g}$ de las mismas.

\section{Adición de plata a las nanopartículas. Formación de nanocompuesto (NC) $\mathrm{Fe}_{3} \mathrm{O}_{4} / \mathrm{Ag}$}

Para la adsorción de plata sobre las NPs se utilizó la técnica descrita por Ghaseminezhada y Shojaosadat (2016). Las nanopartículas de $\mathrm{Fe}_{3} \mathrm{O}_{4}$ fueron suspendidas en $50 \mathrm{ml}$ de agua destilada y la suspensión fue sometida a ultrasonido con el objetivo de disolver las partículas de almidón presentes y una mejor dispersión de las mismas. Luego se añadieron 0,085 g de 
$\mathrm{AgNO}_{3}$ a la solución obteniendo una concentración 0,01 M y una relación másica de 1:1 entre las nanopartículas y la plata. Luego se agregó gota a gota $\mathrm{NH}_{3} \cdot \mathrm{H}_{2} \mathrm{O} 0,8 \mathrm{M}$ hasta alcanzar un $\mathrm{pH}$ igual a 11. La mezcla fue agitada durante una hora con un agitador a $1.000 \mathrm{rpm}$ a $80{ }^{\circ} \mathrm{C}$ para finalmente ser separada por magnetismo a través de imanes y lavada con agua destilada en reiteradas oportunidades.

Luego de añadir el nitrato de plata, se lavó la muestra con agua destilada, llevándola a un sonicador de ultrasonido y poniendo la misma en una base de imanes para producir la separación magnética, y se obtuvo un resultado final de $112,39 \mathrm{mg}$ de NC. Finalmente, las partículas se secaron a temperatura ambiente en un desecador utilizando una bomba de vacío para este proceso, junto con partículas de silica gel (absorbente de humedad del recipiente).

\subsubsection{Caracterización}

La concentración de Fe y Ag fueron medidas por AA (Fig. 4) para lo cual se preparó una solución madre $10 \mathrm{mg} / \mathrm{l}$ en $\mathrm{HNO}_{3}$ al $5 \% \mathrm{v} / \mathrm{v}$, a partir de una solución estándar comercial de 1000 mg/l. La misma se utilizó para preparar soluciones patrones de 0,1 - 0,2 - 0,5 - 1,0 mg/l con los cuales se obtuvieron las curvas de calibración (Fig. 5) utilizadas para determinar la concentración de los respectivos metales en el nanocompuesto. Todas las medidas se realizaron con flujo de aire - acetileno.

Para la determinación de los metales se pesó $10 \mathrm{mg}$ del nanocompuesto, el cual posteriormente fue suspendido en $1000 \mathrm{ml}$ de ácido nítrico al $5 \% \mathrm{v} / \mathrm{v}$ y sometido a ultrasonido para una mejor distribución durante 5 min previos a la medida. La determinación de Ag se realizó a 328,1 nm y la de Fe a 248,3 nm (procedimiento realizado por triplicado)

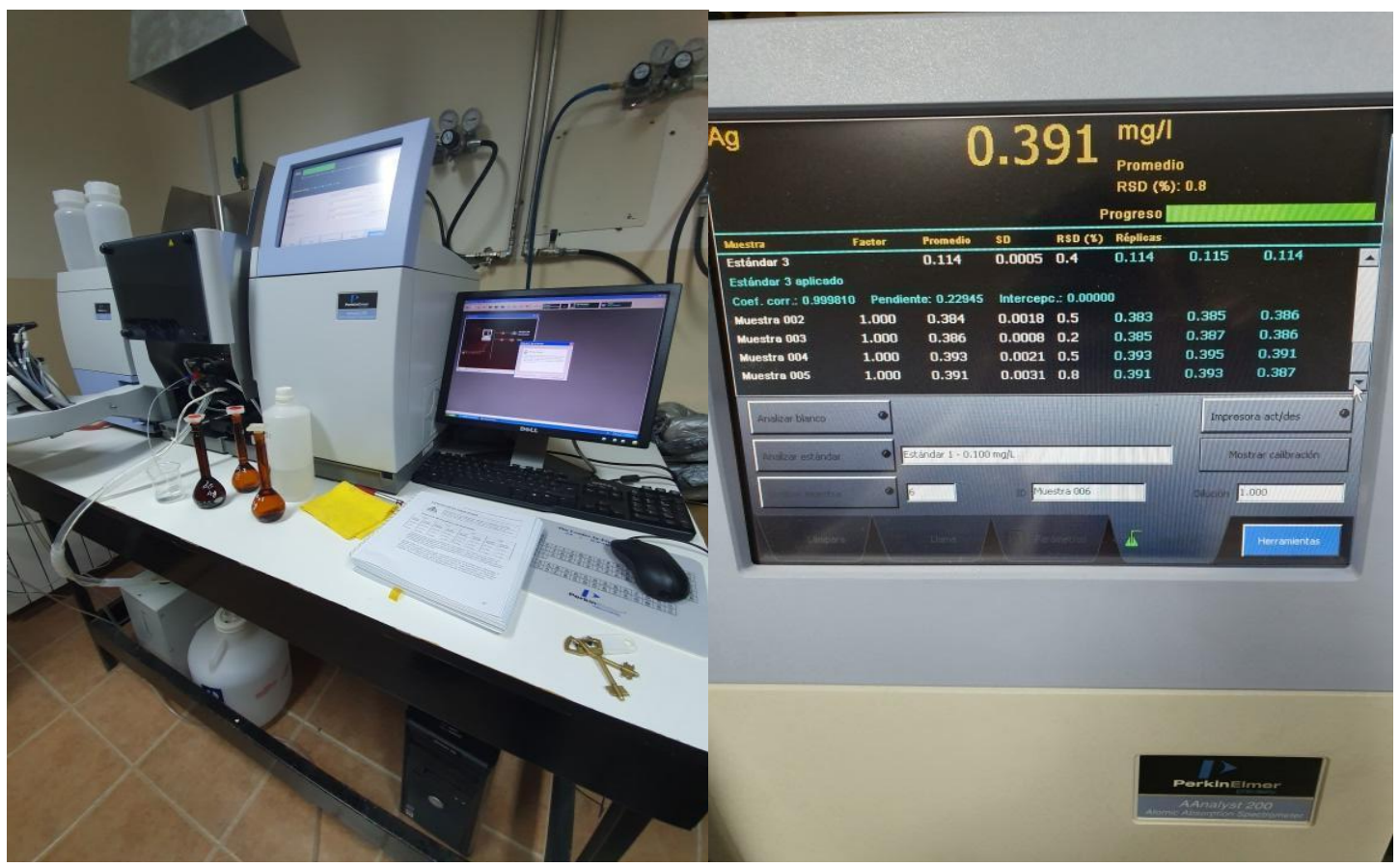

Fig. 4. Concentraciones de Fe y Ag medidas en Espectrofotómetro de absorción atómica Perkin Elmer AAnalyst 200 


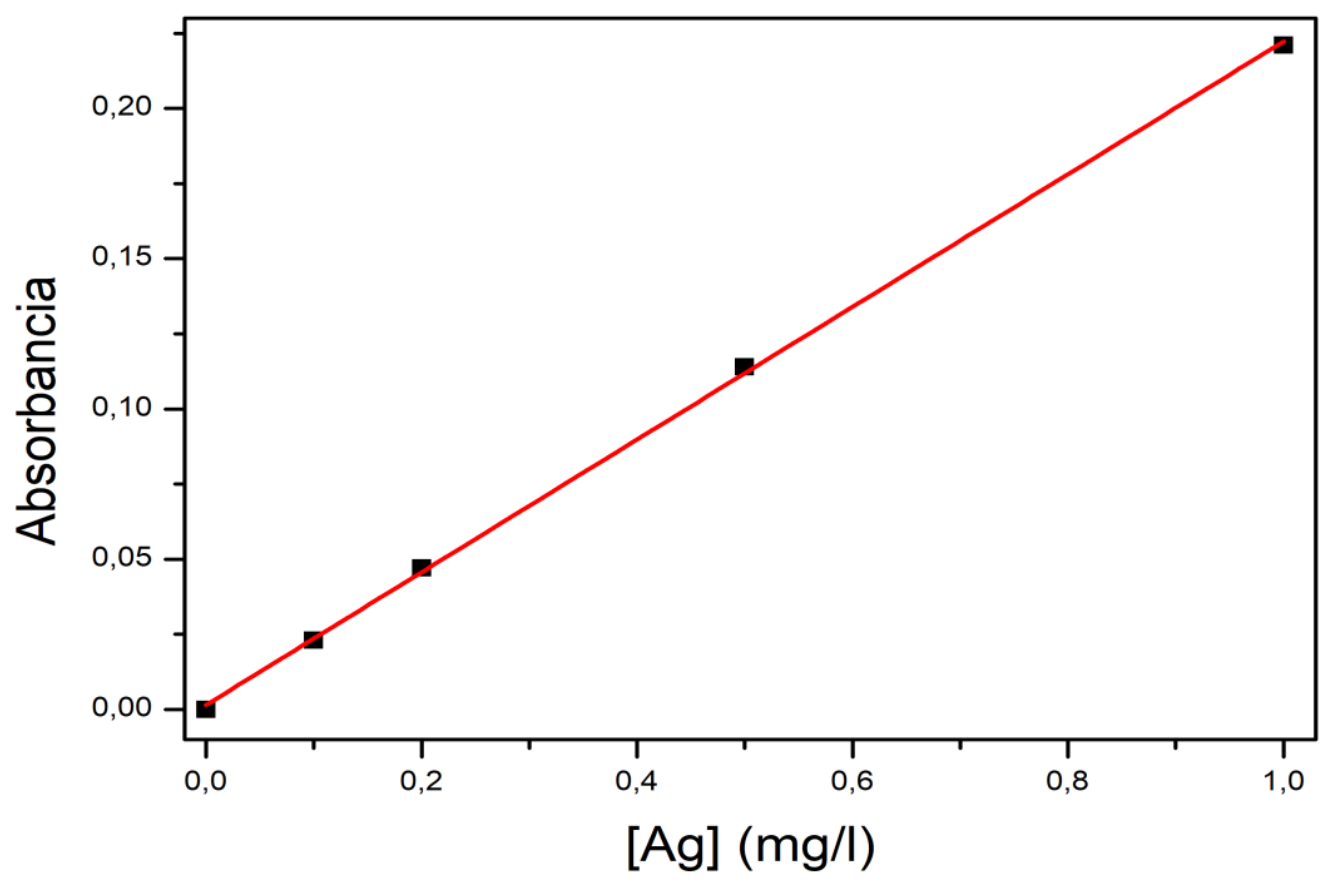

Fig. 5. Curva de calibración para la determinación de Ag por AA

Los valores promedios obtenidos fueron $0,324 \mathrm{mg} / \mathrm{l}$ de Fe y $0,144 \mathrm{mg} / \mathrm{l}$ de $\mathrm{Ag}$ lo que equivale a $32,4 \mathrm{mgFe} / \mathrm{g} \mathrm{NC}$ y $14,4 \mathrm{mg} \mathrm{Ag} / \mathrm{g} \mathrm{NC}$.

\subsubsection{Evaluación del NC como fotosensibilizador}

EL espectro de absorción del nanocompuesto sintetizado muestra absorción de luz en el rango visible entre 400 y $600 \mathrm{~nm}$ aproximadamente (Fig. 6)

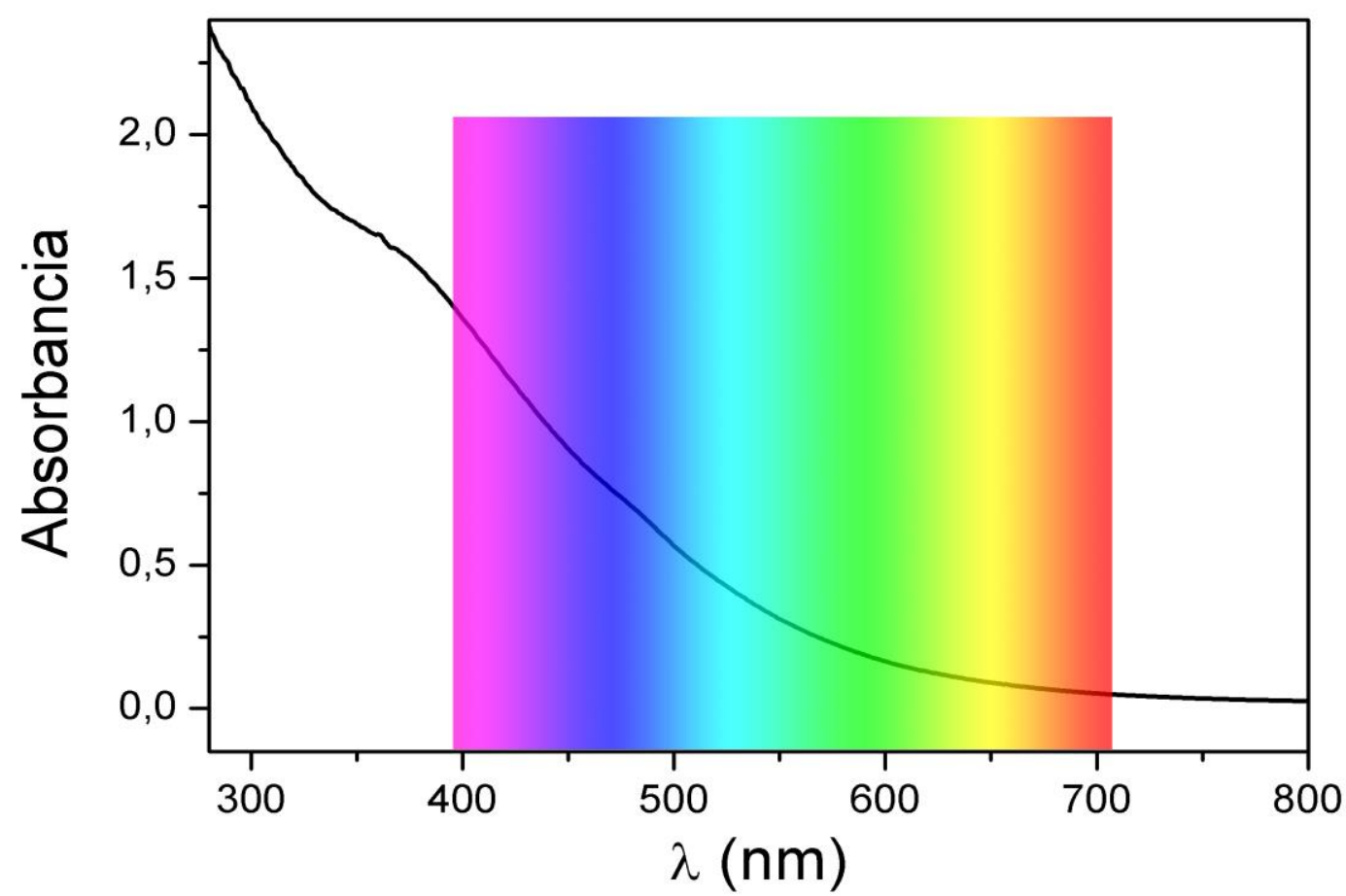

Fig. 6: Espectro de absorción de NC 0,1 mg/l en agua destilada. Los colores representan el espectro de radiación de luz visible 
Para evaluar la posible generación de ROS, se prepararon suspensiones de $19 \mathrm{mg} / \mathrm{l} \mathrm{de} \mathrm{NC}$ en presencia de alcohol furfurílico $5 \times 10^{-4} \mathrm{M}$ como sustrato oxidable de reacción conocida. Las muestras se irradiaron en un fotolizador led con longitud de onda máxima de emisión de $455 \pm 20 \mathrm{~nm}$ (Fig. 7) evaluando la variación de la concentración de $\mathrm{O}_{2}$ durante la fotólisis se observó un leve consumo del mismo ver Fig. 8

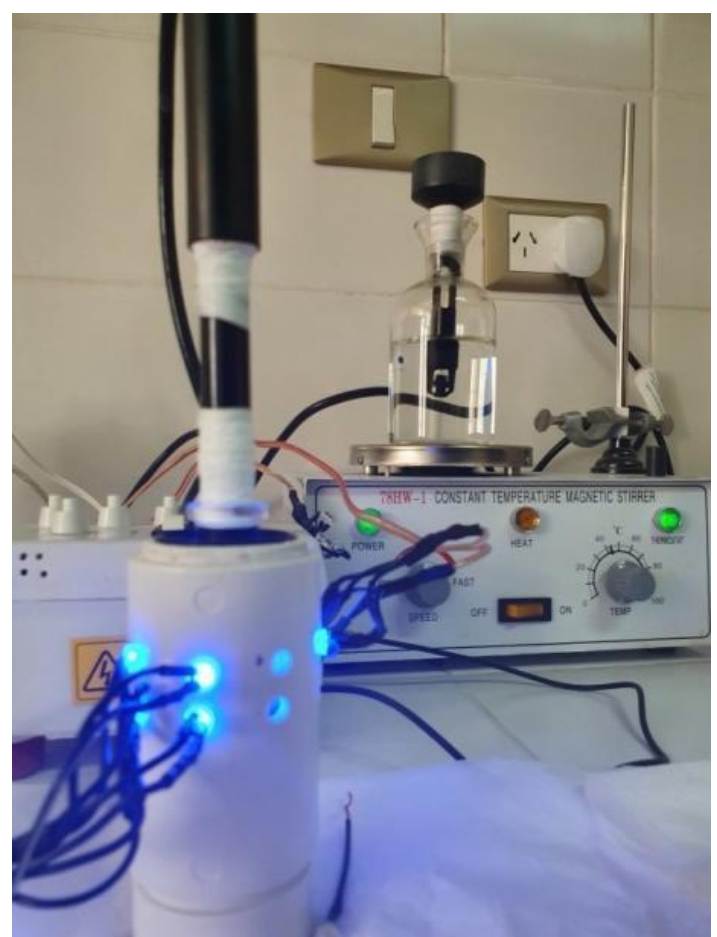

Fig. 7: Fotolizador LED para el análisis del consumo de oxígeno en reacciones fotosensibilizadas

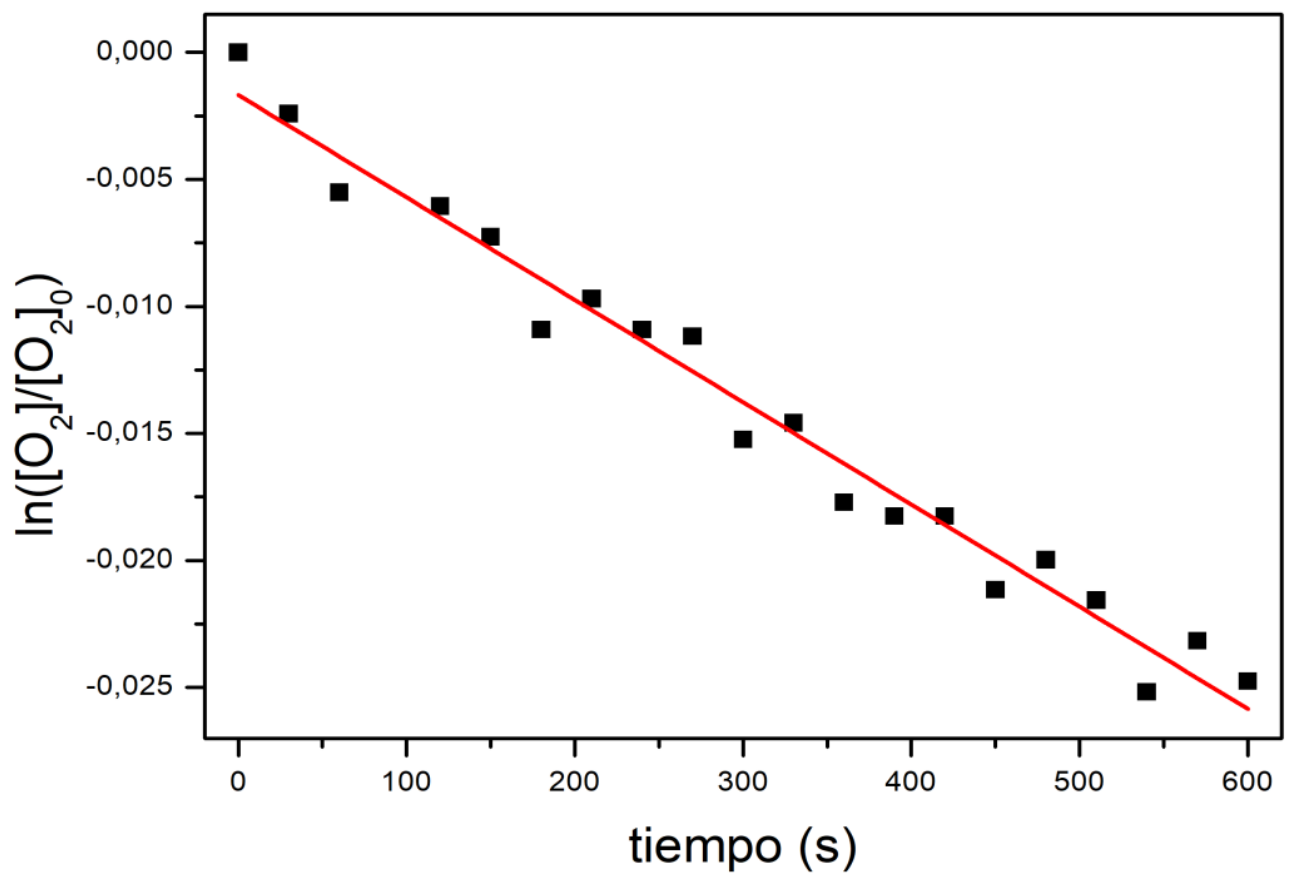

Fig. 8: Gráfico de primer orden para el consumo de oxígeno en la fotólisis de FFA $5 \times 10^{-4} \mathrm{M}$ en presencia de 19 $\mathrm{mg} / \mathrm{l}$ de $\mathrm{NC}$ 
En paralelo se realizaron medidas espectroscópicas de la fotólisis sensibilizada de FFA en presencia del $\mathrm{NC}$ en un fotolizador led, con longitud de onda máxima de emisión de $455 \pm 20$ nm. Éste se insertó en el espectrofotómetro (Fig. 9), para dar lugar luego a la fotólisis, que se realizó con luz led azul durante 20 minutos en intervalos de 1 minuto.

La Fig. 10 muestra la degradación del FFA, pero como se observa en el rango de valores de absorbancia, la misma no fue de gran significancia.

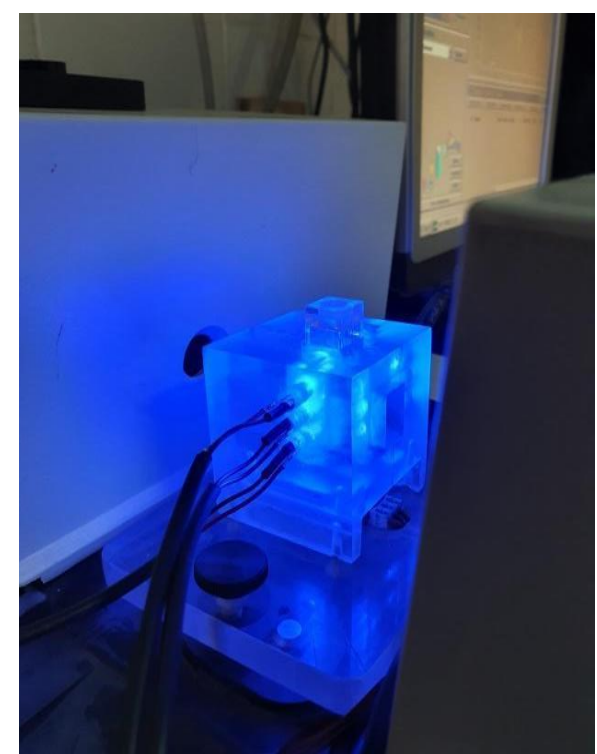

Fig. 9: Fotolizador LED para el análisis del consumo de sustrato en reacciones fotosensibilizadas

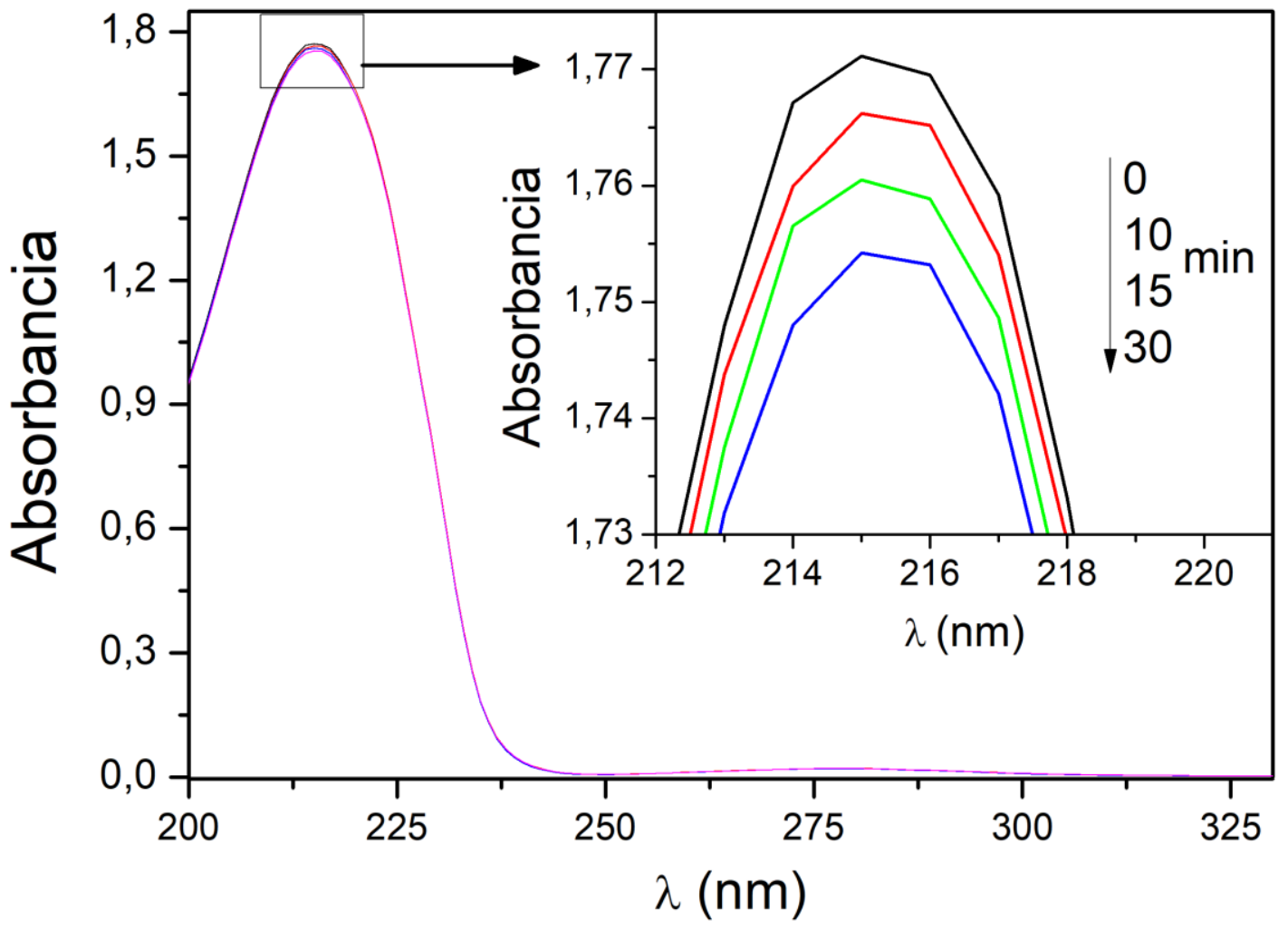

Fig. 10: Cambios en el espectro de absorción UV-vis de una solución acuosa de FFA $\left(5 \times 10^{-4} \mathrm{M}\right)$ para la fotólisis sensibilizada con NC (19 mg/l). Los números en los espectros representan los tiempos de irradiación en minutos. 


\section{CONCLUSIONES}

- De la síntesis del NC y su caracterización

El proceso de síntesis fue satisfactorio obteniendo las partículas deseadas con sus propiedades magnéticas características, aunque se observa un bajo porcentaje de Fe y Ag en relación con la masa total, $3,24 \%$ y $1,44 \%$ respectivamente. Hecho que denota la presencia de gran cantidad de almidón en la matriz del NC. El mismo podría reducirse aumentando la cantidad de lavados o realizando éstos a una mayor temperatura.

- De la fotogeneración de ROS

El NC mostró absorción en el rango visible y generación de ROS que pueden degradar contaminantes ambientales o industriales en solución, aunque con una baja eficiencia, hecho que puede atribuirse a la baja concentración de Ag en el nanocompuesto.

La posibilidad de lograr una separación magnética del fotocatalizador confiere al sistema una eliminación rápida y efectiva del mismo, con la posibilidad de poder reutilizarlo.

\section{REFERENCIAS}

AUFFAN, M.; ROSE, J.; WIESNER, M. R.; BOTTERO, J.-Y. (2009). Chemical Stability of Metallic Nanoparticles: A Parameter Controlling Their Potential Cellular Toxicity in $\begin{array}{lllll}\text { Vitro. } & \text { Environmental } & \text { Pollution, } & 157 & \text { (4), }\end{array}$ https://doi.org/10.1016/j.envpol.2008.10.002.

BOULE, P.; BAHNEMANN, D. (1999). Environmental Photochemistry; Springer: Berlin, Heidelberg; Vol. 2 / 2L.

CHANG, P. R.; YU, J.; MA, X.; ANDERSON, D. P. (2011). Polysaccharides as Stabilizers for the Synthesis of Magnetic Nanoparticles. Carbohydrate Polymers, 83 (2), 640644. https://doi.org/10.1016/j.carbpol.2010.08.027

CHEN, J.; YANG, L.; CHEN, J.; LIU, W.; ZHANG, D.; XU, P.; DAI, T.; SHANG, L.; YANG, Y.; TANG, S.; ZHANG, Y.; LIN, H.; CHEN, Z.; HUANG, M. (2019). Composite of Silver Nanoparticles and Photosensitizer Leads to Mutual Enhancement of Antimicrobial Efficacy and Promotes Wound Healing. Chemical Engineering Journal, 374, 1373-1381. https://doi.org/10.1016/j.cej.2019.05.184

GHASEMINEZHAD, S. M.; SHOJAOSADATI, S. A. (2016). Evaluation of the Antibacterial Activity of $\mathrm{Ag} / \mathrm{Fe} 3 \mathrm{O} 4$ Nanocomposites Synthesized Using Starch. Carbohydrate Polymers, 144, 454-463. https://doi.org/10.1016/j.carbpol.2016.03.007

HALliWELL, B. (2006). Free Radicals in Biochemistry and Medicine. In Reviews in Cell Biology and Molecular Medicine; American Cancer Society. https://doi.org/10.1002/3527600906.mcb.200300036

HORIKOSHI, S.; SERPONE, N. (2013). Introduction to Nanoparticles. In Microwaves in Nanoparticle Synthesis; John Wiley \& Sons, Ltd; pp 1-24. https://doi.org/10.1002/9783527648122

LAURENT, S.; FORGE, D.; PORT, M.; ROCH, A.; ROBIC, C.; VANDER ELST, L.; MULLER, R. N. (2008). Magnetic Iron Oxide Nanoparticles: Synthesis, Stabilization, Vectorization, Physicochemical Characterizations, and Biological Applications. Chem. Rev., 108 (6), 2064-2110. https://doi.org/10.1021/cr068445e 
LIU, X.; LI, X.; LIU, X.; HE, S.; JIN, J.; MENG, H. (2020). Green Preparation of Ag-ZnORGO Nanoparticles for Efficient Adsorption and Photodegradation Activity. Colloids and Surfaces A: Physicochemical and Engineering Aspects, 584, 124011. https://doi.org/10.1016/j.colsurfa.2019.124011

LIU, T.; WANG, C.; WANG, W.; YANG, G.; LU, Z.; XU, P.; SUN, X.; ZHANG, J. (2021). The Enhanced Properties in Photocatalytic Wastewater Treatment: Sulfanilamide (SAM) Photodegradation and Cr6+ Photoreduction on Magnetic $\mathrm{Ag} / \mathrm{ZnFe} 2 \mathrm{O} 4$ Nanoarchitectures. Journal of Alloys and Compounds, 867, 159085. https://doi.org/10.1016/j.jallcom.2021.159085

SCHULTE, J.; DUTTA, J. (2005). Nanotechnology in Environmental Protection and Pollution. Science and Technology of Advanced Materials, 6 (3), 219-220. https://doi.org/10.1016/j.stam.2005.03.009

WANG, Y.; GAO, P.; WEI, Y.; JIN, Y.; SUN, S.; WANG, Z.; JIANG, Y. (2021). Silver Nanoparticles Decorated Magnetic Polymer Composites (Fe3O4@PS@Ag) as Highly Efficient Reusable Catalyst for the Degradation of 4-Nitrophenol and Organic Dyes. Journal of Environmental Management, 278, 111473. https://doi.org/10.1016/j.jenvman.2020.111473 